\title{
The neurobiology of cell death in glaucoma
}

\begin{abstract}
Glaucoma is an optic neuropathy in which the optic nerve axons are damaged, resulting in death of retinal ganglion cells (RGCs). The primary region of damage is thought to be the optic nerve head (ONH), with the lateral geniculate nucleus (LGN) and optic radiations to the visual cortex being secondarily affected. Neurotrophin deprivation resulting from optic nerve injury is thought to cause RGCs to die by apoptosis by inhibition of cell survival pathways. However, disruption of retrograde axonal transport is not the only mechanism associated with optic nerve damage and RGC death, and thus, an additional mechanism of injury is likely to be involved in glaucomatous optic neuropathy.

Eye (2007) 21, S11-S14; doi:10.1038/sj.eye.6702880
\end{abstract}

Keywords: glaucoma; optic neuropathy; retinal ganglion cells; oxonal injury

\section{Introduction}

Historically, glaucoma has been described as a disease caused by increased ocular pressure (IOP). Only recently has glaucoma been considered a primary optic neuropathy, but this fact is not widely shared by the public and health-care professionals alike. An informal survey of mainstream dictionary definitions of glaucoma still reveals obsolete phrases such as 'increased intraocular pressure that results in...' and 'a group of eye diseases characterized by pressure that is too high for the optic nerve to withstand'. Many eye care professionals continue to communicate these false notions as well, perhaps as a more efficient way to explain the disease process to patients and thereby improve compliance with IOP-lowering medicines. While this simplification theoretically helps facilitate patient-physician communication about glaucoma, it is nonetheless inaccurate. However, there is

JP Vrabec ${ }^{1}$ and LA Levin ${ }^{1,2}$

abundant evidence demonstrating that ocular hypertension alone is neither a sufficient nor a necessary factor for the development or progression of glaucoma. In each of the major, randomized clinical glaucoma trials (Advanced Glaucoma Intervention Study (AGIS), ${ }^{1,2}$

Collaborative Normal Tension Glaucoma Study (CNTGS), ${ }^{3,4}$ Collaborative Initial Glaucoma

Treatment Study (CIGTS), ${ }^{5}$ and Early Manifest Glaucoma Trial $(\text { EMGT })^{6}$ ), there was documented progression of disease despite a significant lowering of IOP, and there are many examples of continued glaucomatous progression despite IOP as low as 7 or $8 \mathrm{mmHg}$. Conversely, many patients have ocular hypertension for years without functional or structural loss of retinal ganglion cells (RGCs) and their axons.

The Cochrane Eyes and Vision Group defines glaucoma as 'a disease characterized by defects in the visual field, damage to the nerve at the back of the eye, and usually raised pressure inside the eye.' As we now know, this view of glaucoma as an optic neuropathy with elevated IOP as a modifiable risk factor rather than as the causative agent for damage is more correct, although it still does not take into account the characteristic morphological and functional aspects of the disease. It is important for clinicians and the research community alike to view glaucoma as an optic neuropathy, especially as we drive toward discovering better therapeutic strategies, including pharmacologic neuroprotection. This review will discuss the nuances of glaucoma as an optic neuropathy, compare it to other optic neuropathies, and visit the neurobiological mechanisms of cell death in glaucoma.

\section{Glaucoma and other optic neuropathies as axonal diseases}

We believe that glaucoma is best described as an optic neuropathy with highly characteristic changes in optic nerve head $(\mathrm{ONH})$
'Department of Ophthalmology and Visual Sciences, University of Wisconsin Medical School, Madison, WI, USA

${ }^{2}$ Department of Ophthalmology, University of Montréal, Montréal, Canada

Correspondence: LA Levin, Department of Ophthalmology and Visual Sciences, University of Wisconsin Medical School, 600 Highland Avenue, Madison,

WI 53792, USA Tel: +1 16082656546 . 
morphology, accompanied by thinning of the nerve fibre layer, and eventual loss of RGCs. These changes result in corresponding visual field deficits that, without treatment, can eventually progress to complete vision loss. RGCs are known from animal and human studies to undergo cell death by apoptosis after axonal injury. ${ }^{7,8}$ This cell death and simultaneous loss of RGC axons is thought to be the cause of the excavated appearance of the ONH in glaucoma, ${ }^{9}$ although it is still unclear why there is so much cupping in glaucoma compared to other diseases of RGC axons, for example nonarteritic anterior optic neuropathy, traumatic optic neuropathy, and so on. Nonetheless, axonal damage is likely the primary site of injury in glaucoma. This is supported by previous visual field studies ${ }^{10,11}$ that showed that the primary field deficits in glaucoma occur in a nerve fibre bundle pattern reflecting optic disc changes. Likewise, in ischaemic optic neuropathy, the most common acute optic neuropathy in older individuals, the damage clearly occurs at the axonal level, specifically in the retrolaminar region. Compressive and traumatic optic neuropathies, as well as optic neuritis in multiple sclerosis, are also always axonal processes, which paradoxically rarely end up with $\mathrm{ONH}$ excavation.

\section{Sites of damage along the visual pathway}

The human optic nerve is comprised of approximately 1.2 million RGC axons. These central nervous system (CNS) axons originate at the RGC cell bodies within the neural retina, travel within the nerve fibre layer posterior toward the optic disc, and then turn sharply at the disc through the lamina cribrosa to form the optic nerve, gaining their myelin sheath in the process. Visual information entering the eye is processed in the retina and transmitted via these axons to the lateral geniculate nucleus (LGN), from where signals are relayed to the visual cortex. Other axons project to the superior colliculus (for orienting responses), the suprachiasmatic nucleus (for light entrainment of the sleep-wake cycle), and the pretectal nuclei (for the pupillary light reaction). In glaucoma, the primary region of damage is thought to be at the immediate peripapillary retina, disk, or anterior lamina cribrosa, most likely a consequence of the axons' specific vulnerability as they make the turn through this zone. Additionally, the LGN, optic radiations, and the visual cortex may also be affected in glaucoma. ${ }^{12,13}$ While it is not exactly clear whether this posterior involvement is due to primary or secondary damage, it is most likely a secondary effect, as visual field deficits from LGN injuries appear quite different from those observed in glaucoma.

\section{Role of neurotrophin deprivation in RGC death}

Clues to the neurobiology of axonal injury can be found within the realm of developmental biology. During embryonic development, RGCs extend neurites to their targets in the brain, which are actively secreting specific neurotrophins, most importantly brain-derived neurotrophic factor (BDNF). Within the developing retina, there is an excess of RGCs competing for these target-derived neurotrophins. Those RGCs that do not obtain sufficient neurotrophins eventually die by apoptosis. ${ }^{14,15}$ Other factors are important for RGC survival, including neurotrophin 4/5 (NT4/5), ciliary neurotrophic factor (CNTF), insulin-like growth factor-1 (IGF-1), and many others.

In adulthood, a similar communication likely takes place between RGCs and their CNS targets, as the same target-derived neurotrophins are continuously transported in retrograde fashion from the brain back to the RGC cell bodies. In the absence of these factors, so-called neurotrophin deprivation, RGCs begin to undergo apoptosis (Figure 1). It is likely that disruption of the supply of neurotrophins plays a major role in the axonal loss of glaucoma. One possible mechanism would be inhibition of retrograde transport by ischaemia or increased IOP, ${ }^{16}$ resulting in neurotrophin deprivation, along with the loss of the visual signal (Figure 1). It has also been shown that, in glaucoma, the blockade of retrograde transport of BDNF and its receptor TrkB will co-occur in the retrolaminar area. ${ }^{17,18}$

Irrespective of mechanism, axonal injury triggers the death of RGCs. While some believe that this leads to pathologically significant release of glutamate and adjacent RGC death by secondary degeneration, there is

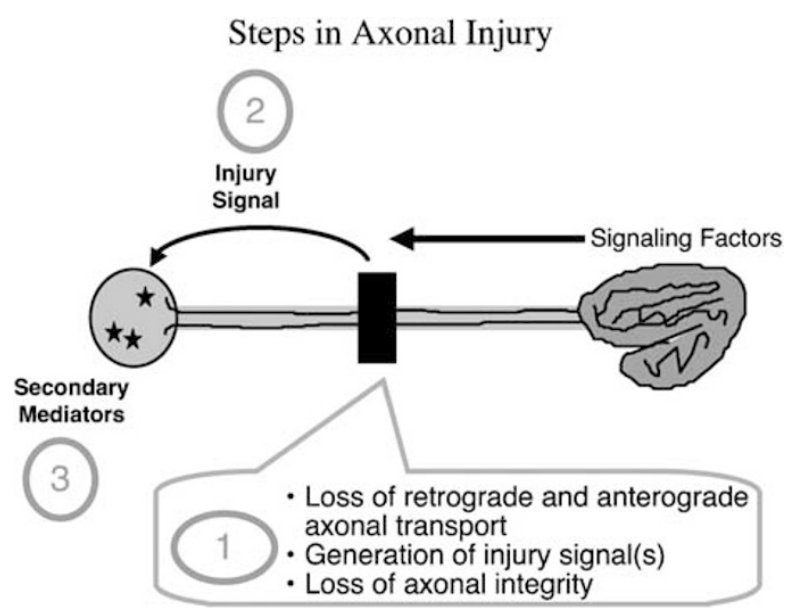

Figure 1 Mechanisms involved in the blockage of retrograde transport of growth factors through ischaemia or increased intraocular pressure. (Reproduced with permission from L Levin.) 
reason to believe that this does not occur. ${ }^{19}$ Eventually there is Wallerian degeneration of the axon and permanent loss of visual function. These end stages are seen clinically in glaucoma as loss of the retinal nerve fibre layer and visual field deficits. ${ }^{20-22}$

As described above, there are a large number of neurotrophic factors, of which the most important is BDNF. The signalling pathways of BDNF have been well characterized. BDNF binds the TrkB receptor, which activates the phosphoinositide 3 (PI3)-kinase or mitogenactivated protein (MAP) kinase cascades. The MAP kinases most likely ${ }^{23}$ provide further downstream signalling, which results in the maintenance of cell survival. The retina itself produces $\mathrm{BDNF}^{24-26}$ and RGCs will survive for longer if they are maintained with BDNF injections into the vitreous, although the effects are temporary so they still do not survive for very long. ${ }^{27}$

There is no definitive evidence as to whether neurotrophin deprivation is the only cause of RGC death after axotomy or axonal injury. RGC survival in the presence of neurotrophic factor does not mean that these cells are dependent on neurotrophic factor, only that it is sufficient for their survival. Clinical situations are known in which RGCs survive despite the longstanding loss or reduction in axonal transport, for example papilledema. Moreover, the rate of axonal transport loss is much faster than the rate of RGC death after axonal injury, and changes in responsiveness to neurotrophic factors are independent of neurotrophin levels themselves. For example, levels of the BDNF receptor decrease despite sufficient neurotrophic factor, resulting from decreased levels of cyclic AMP that are independent of the presence of growth factor itself. ${ }^{28}$ Even prolonged delivery of BDNF and its TrkB receptor will ultimately fail to keep RGCs alive. ${ }^{27}$

A further complication is that we have incomplete knowledge of the complexity of the MAP kinase signalling pathways, particularly with respect to differences between target-mediated and cell bodymediated neurotrophin signalling. It is possible that the retrograde Erk5 pathway is crucial for RGC survival, ${ }^{29}$ but this has not yet been studied. Further understanding of these and other signalling pathways should provide helpful additional context for the interaction of neurotrophins, RGC death, and glaucoma.

\section{Conclusions}

We argue that neurotrophin deprivation alone is not sufficient as an explanation of RGC death. However, several areas remain poorly understood and questions remain. Does it make a difference whether we treat the axon terminal in the brain or at the cell body? Other than causing neurotrophin deprivation, what other pathways does axonal transport blockade or axonal injury affect? Does neuroprotection without protection of the axon ('axoprotection') have utility for clinical disease? For neuroprotective strategies based on neurotrophins to be developed fully, we suggest that these fundamental questions should be addressed.

\section{Acknowledgements}

Grant support: This work was supported by Grants NIH R01EY12492 and P30EY016665, Retina Research Foundation, and an unrestricted departmental grant from Research to Prevent Blindness Inc.

\section{References}

1 The AGIS Investigators. The advanced glaucoma intervention study, 6: effect of cataract on visual field and visual acuity. The AGIS Investigators. Arch Ophthalmol 2000; 118: 1639-1652.

2 The AGIS Investigators. The Advanced Glaucoma Intervention Study (AGIS): 1. Study design and methods and baseline characteristics of study patients. Control Clin Trials 1994; 15: 299-325.

3 Collaborative Normal-Tension Glaucoma Study Group. The effectiveness of intraocular pressure reduction in the treatment of normal-tension glaucoma. Collaborative Normal-Tension Glaucoma Study Group. Am J Ophthalmol 1998; 126: 498-505.

4 Collaborative Normal-Tension Glaucoma Study Group. Comparison of glaucomatous progression between untreated patients with normal-tension glaucoma and patients with therapeutically reduced intraocular pressures. Collaborative Normal-Tension Glaucoma Study Group. Am J Ophthalmol 1998; 126: 487-497.

5 Lichter PR, Musch DC, Gillespie BW, Guire KE, Janz NK, Wren PA. Interim clinical outcomes in the Collaborative Initial Glaucoma Treatment Study comparing initial treatment randomized to medications or surgery. Ophthalmology 2001; 108: 1943-1953.

6 Heijl A, Leske MC, Bengtsson B, Hyman L, Hussein M. Reduction of intraocular pressure and glaucoma progression: results from the Early Manifest Glaucoma Trial. Arch Ophthalmol 2002; 120: 1268-1279.

7 Berkelaar M, Clarke DB, Wang YC, Bray GM, Aguayo AJ. Axotomy results in delayed death and apoptosis of retinal ganglion cells in adult rats. J Neurosci 1994; 14(7): 4368-4374.

8 Lieven CJ, Hoegger MJ, Schlieve CR, Levin LA. Retinal ganglion cell axotomy induces an increase in intracellular superoxide anion. Invest Ophthalmol Vis Sci 2006; 47(4): 1477-1485.

9 Quigley HA, Green WR. The histology of human glaucoma cupping and optic nerve damage: clinicopathologic correlation in 21 eyes. Ophthalmology 1979; 86(10): 1803-1830.

10 Boden C, Sample PA, Boehm AG, Vasile C, Akinepalli R, Weinreb RN. The structure-function relationship in eyes with glaucomatous visual field loss that crosses the horizontal meridian. Arch Ophthalmol 2002; 120: 907-912. 
11 Levin LA. Relevance of the site of injury of glaucoma to neuroprotective strategies. Surv Ophthalmol 2001; 45(Suppl 3): S243-S249. discussion S273-S276.

12 Yucel YH, Zhang Q, Gupta N, Kaufman PL, Weinreb RN. Loss of neurons in magnocellular and parvocellular layers of the lateral geniculate nucleus in glaucoma. Arch Ophthalmol 2000; 118(3): 378-384.

13 Malpeli JG, Lee D, Baker FH. Laminar and retinotopic organization of the macaque lateral geniculate nucleus: magnocellular and parvocellular magnification functions. J Comp Neurol 1996; 375(3): 363-377.

14 Meyer-Franke A, Kaplan MR, Pfrieger FW, Barres BA. Characterization of the signaling interactions that promote the survival and growth of developing retinal ganglion cells in culture. Neuron 1995; 15(4): 805-819.

15 Nickells RW. Retinal ganglion cell death in glaucoma: the how, the why, and the maybe. J Glaucoma 1996; 5(5): 345-356.

16 Minckler DS, Bunt AH, Johanson GW. Orthograde and retrograde axoplasmic transport during acute ocular hypertension in the monkey. Invest Ophthalmol Vis Sci 1977; 16(5): 426-441.

17 Johnson EC, Deppmeier LMH, Wentzien SKF, Hsu I, Morrison JC. Chronology of optic nerve head and retinal responses to elevated intraocular pressure. Invest Ophthalmol Vis Sci 2000; 41: 431-442.

18 Pease ME, McKinnon SJ, Quigley HA, Kerrigan-Baumrind LA, Zack DJ. Obstructed axonal transport of BDNF and its receptor TrkB in experimental glaucoma. Invest Ophthalmol Vis Sci 2000; 41: 764-774.

19 Levin LA. Relevance of the site of injury of glaucoma to neuroprotective strategies. Surv Ophthalmol 2001; 45: S243-S249.

20 Kerrigan LA, Zack DJ, Quigley HA, Smith SD, Pease ME. TUNEL-positive ganglion cells in human primary openangle glaucoma. Arch Ophthalmol 1997; 115(8): 1031-1035.
21 Okisaka S, Murakami A, Mizukawa A, Ito J. Apoptosis in retinal ganglion cell decrease in human glaucomatous eyes. Jpn J Ophthalmol 1997; 41(2): 84-88.

22 Tuulonen A, Airaksinen PJ. Initial glaucomatous optic disk and retinal nerve fiber layer abnormalities and their progression. Am J Ophthalmol 1991; 111(4): 485-490.

23 Pernet V, Hauswirth WW, Di Polo A. Extracellular signal-regulated kinase $1 / 2$ mediates survival, but not axon regeneration, of adult injured CNS neurons in vivo. J Neurochem 2005; 93: 72-83.

24 Cellerino A, Kohler K. Brain-derived neurotrophic factor/ neurotrophin-4 receptor TrkB is localised on ganglion cells and dopaminergic amacrine cells in the vertebrate retina. J Comp Neurol 1997; 386(1): 149-160.

25 Herzog KH, von Bartheld CS. Contributions of the optic tectum and the retina as sources of brain-derived neurotrophic factor for retinal ganglion cells in the chick embryo. J Neurosci 1998; 18(8): 2891-2906.

26 Perez MT, Caminos E. Expression of brain-derived neurotrophic factor and of its functional receptor in neonatal and adult rat retina. Neurosci Lett 1995; 183(1-2): 96-99.

27 Di Polo A, Aigner LJ, Dunn RJ, Bray GM, Aguayo AJ. Prolonged delivery of brain-derived neurotrophic factor by adenovirus-infected Muller cells temporarily rescues injured retinal ganglion cells. Proc Natl Acad Sci USA 1998; 95(7): 3978-3983.

28 Meyer-Franke A, Wilkinson GA, Kruttgen A, Hu M, Monro E, Hanson MG et al. Depolarization and cAMP elevation rapidly recruit TrkB to the plasma membrane of CNS neurons. Neuron 1998; 21: 681-693.

29 Watson FL, Heerssen HM, Bhattacharyya A, Klesse L, Lin MZ, Segal RA. Neurotrophins use the Erk5 pathway to mediate a retrograde survival response. Nat Neurosci 2001; 4: 981-988. 